\title{
Welcome message from the Editor-in-Chief
}

\author{
Phan Cong Vinh \\ Faculty of Information Technology at Nguyen Tat Thanh University, Ho Chi Minh City, \\ Vietnam
}

\begin{abstract}
Received on 28 July 2015; published on 04 August 2015
Copyright (C) 2015 Phan Cong Vinh, licensed to ICST. This is an open access article distributed under the terms of the Creative Commons Attribution license (http://creativecommons.org/licenses/by/3.0/), which permits unlimited use, distribution and reproduction in any medium so long as the original work is properly cited.

doi:10.4108/eai.4-8-2015.150040
\end{abstract}

On behalf of the Editorial board, we welcome you to the fourth issue of the ICST Transactions on ContextAware Systems and Applications. In this issue, we present four selected papers that span various aspects of context-aware systems and applications, and an introduction to the book titled "Nature of Computation and Communication".

This issue will serve as a reference material for researchers, scientists, professionals and students in computer science and computer engineering as well as developers and practitioners in computing and networking systems design by providing them with state-of-the-art research findings and future opportunities and trends. These papers include some recent advances in context-awareness reflected in this issue. In particular, the issue covers various themes of context-awareness as follows:

Paper 1 by N. Khabou, I. B. Rodriguez and M. Jmaiel reports that the increase of mobile and interconnected devices leads to the growth of demands for context aware applications. These applications deployed on top of pervasive environments must adapt themselves to context changes. Context aware applications have to continuously sense their physical environment, and adapt their behavior accordingly. These applications must perform four phases starting by collecting and monitoring context, then analyzing context, deciding adaptation actions and finally executing the planned adaptation actions to deal with the context changes. In this paper, authors focus on the second phase. Authors propose a context analysis approach that relies on different thresholds defined according to the user needs to detect context changes and raise notifications when changes occur. The analysis approach is performed according to three different steps. A context storage

*Corresponding author. Email: pcvinh@ntt.edu.vn step, a context classification step, and a threshold calculation step.

Paper 2 by N.K. Quoc, V.T. Tu and N.T. Hai reports that the congestion control by the active queue management mechanism at the network nodes is essential. In recent years, some researchers have taken computational intelligence, especially in fuzzy reasoning active queue management to enhance network performance. However, the fuzzy controller works depends very much expert and cannot be updated according to changes in the network, so the efficiency of the mechanism is not high. Therefore, authors propose an integrated model of fuzzy reasoning and neural networks (so-called FNN). The results of the training of neural network will optimize the fuzzy controller, adapt to network changes. This will improve the operational efficiency of the mechanism of active queue management at network nodes.

Paper 3 by N.T. Binh proposes a method to implement for human object detection based on context awareness in new wavelet generation domain in outdoor environment. Author uses curvelet transform based on context awareness combined with support vector machines as a classifier for human detection. The proposed method has been tested on a standard dataset like PEST2001 dataset. For demonstrating the superiority of the proposed method, author has compared the results with the other recent methods available in literature.

Paper 4 by N. T. Tung introduces some highlighted activities of the Fourth International Conference on Context-Aware Systems and Applications (ICCASA 2015). The ICCASA 2015 is jointly organized by EAI, Nguyen Tat Thanh University (NTTU), and Ba Ria-Vung Tau University (BRVTU) and endorsed by the European Alliance for Innovation (EAI), a leading communitybased organization devoted to the advancement of innovation in the field of ICT. The event consists of a main track on context-aware systems and applications, and two special tracks on contextual recommendation systems and on computational aspects of context in 
natural language processing, respectively. Especially, there are three keynote speeches, which will be presented at ICCASA 2015 by prominent invited speakers

Paper 5 by P. C. Vinh introduces the book with the title of "Nature of Computation and Communication" edited by Prof. Phan Cong Vinh, Dr. Emil Vassev and Prof Mike Hinchey, and published by Springer in 2015 to scientists, researchers, professionals and students in the field of computing. Hopefully, they will find this book provoking for their research related to hot topics of computing and being useful to their future work.

For the preparation of this fourth issue we would like to acknowledge the work of all our Editors, reviewers and authors who have positively supported this publication. We will be happy to receive from our readers any suggestions, including possible proposals for future special issues, which may contribute to further maintain the high scientific quality and relevance of this journal.

We hope you will find this fourth issue provoking for your research in the field of context-awareness and being useful to your future work.

\section{About the Editor-in-Chief}

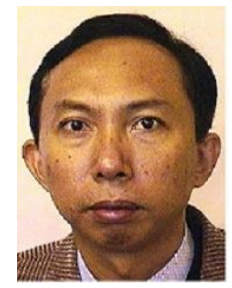

Phan Cong Vinh received a $\mathrm{PhD}$ in computer science from London South Bank University (LSBU) in the United Kingdom, a BS in mathematics and an MS in computer science from Vietnam National University (VNU) in Ho Chi Minh City, and a BA in English from Hanoi University of Foreign Languages Studies in Vietnam.
He finished his PhD dissertation with the title Formal Aspects of Dynamic Reconfigurability in Reconfigurable Computing Systems supervised by Prof. Jonathan P. Bowen at LSBU where he was affiliated with the Centre for Applied Formal Methods (CAFM) at the Institute for Computing Research (ICR). He joined research with Dr. Tomasz Janowski at the International Institute for Software Technology (IIST) in Macao SAR, China, as a fellow in 2000. At present, he is an Associate Professor of Nguyen Tat Thanh University (NTTU) to take on the responsibility of a senior research scientist. He has been author or co-author of many refereed contributions published in prestigious journals, conference proceedings or edited books. He is the author of a book on computing science titled ŞDynamic Reconfigurability in Reconfigurable Computing Systems: Formal Aspects of Computing ̌̃ (VDM, 2009); editor of two titles, ŞAutonomic Networking-on-Chip: BioInspired Specification, Development and Verification (CRC Press, 2012) and ŞFormal and Practical Aspects of Autonomic Computing and Networking: Specification, Development and Verification ̌̌ (IGI Global, 2011); editor of Special Issues, ŞContext-Awareness of Mobile Systems: Models, Algorithms and Applications Ť (Springer MONET, 2012 (indexed in SCIE)) and ŞAdvances in Autonomic Computing: Formal Engineering Methods for Nature-Inspired Computing SystemsŤ (Springer TCS, 2012). He has served on many conference program committees and has been general or technical (co)chair and (co)organizer of several international conferences such as ICCASA and ICTCC. His research interests center on all aspects of formal methods, nature of computation and communication, and applied categorical structures in computer science. 